\title{
Steady-state Voltage Profile and Long-term Voltage Stability of Electrified Road with Wireless Dynamic Charging
}

\author{
Chuan Wang \\ Nanyang Technological University \\ Singapore \\ chuan002@e.ntu.edu.sg
}

\author{
Hung D. Nguyen * \\ Nanyang Technological University \\ Singapore \\ hunghtd@ntu.edu.sg
}

\begin{abstract}
Wireless dynamic charging technologies are becoming a promising alternative solution to plug-in ones as they allow on-the-move charging for electric vehicles. From a power network point of view, this charging makes electric vehicles a new type of loads-the moving loads. Such moving loads differ from the traditional loads, as they may change their locations constantly in the grids. To study the effect of these moving loads on power distribution grids, this work focuses on the steady-state analysis of electrified roads equipped with wireless dynamic charging. In particular, the voltage profile and the long-term voltage stability of the electrified roads are considered. Unusual shapes of the voltage profile are observed such as the half-leaf veins for a one-way road and the harp-like shape for a two-way road. Voltage swings are also detected while the vehicles move in the two-way road configuration. As for the long-term voltage stability, continuation powerfl ow is used to characterize the maximum length of a road and the maximum number of vehicles that the road can accommodate.
\end{abstract}

\section{CCS CONCEPTS}

- Networks $\rightarrow$ Cyber-physical networks; • Computing methodologies $\rightarrow$ Simulation evaluation; • Applied computing $\rightarrow$ Engineering.

\section{KEYWORDS}

Electric vehicle, Wireless dynamic charging (WDC), Moving load

\section{ACM Reference Format:}

Chuan Wang and Hung D. Nguyen . 2019. Steady-state Voltage Profile and Long-term Voltage Stability of Electrified Road with Wireless Dynamic Charging. In Proceedings of the Tenth ACM International Conference on Future Energy Systems (e-Energy '19), fune 25-28, 2019, Phoenix, AZ, USA. ACM, New York, NY, USA, 5 pages. https://doi.org/10.1145/3307772.3328294

\section{INTRODUCTION}

Electric vehicles (EVs) becomes an integral part of urban transportation. They may overtake conventional vehicles with combustion engines soon owing to having advantages making no noise,

${ }^{*}$ This author is the corresponding author of this research.

Permission to make digital or hard copies of part or all of this work for personal or classroom use is granted without fee provided that copies are not made or distributed for profit or commercial advantage and that copies bear this notice and the full citation on thefi rst page. Copyrights for third-party components of this work must be honored

For all other uses, contact the owner/author(s).

e-Energy '19, June 25-28, 2019, Phoenix, AZ, USA

(c) 2019 Copyright held by the owner/author(s).

ACM ISBN 978-1-4503-6671-7/19/06.

https://doi.org/10.1145/3307772.3328294 releasing no pollution at the vehicle level, and having higher wellto-wheels efficiency. EVs can be further classified as stationary and dynamic charging vehicles according to the charging method. Dynamic charging method allows charging in motions so it resolves long charging time in the stationary counterpart. Among dynamic charging technologies, wireless dynamic charging (WDC) is emerging as it can charge vehicles on the move without connecting directly to power cables [1-3].

Currently, the research trends focus on the charging technologies mostly in the device level such as maintaining charging efficiency, regulating battery capacity, and improving economic benefits [4-6]. The effects of dynamic charging at the system level have not been studied extensively. Interestingly, a relevant research area, however, is railway electrification systems with a focus on electric locomotives. Most importantly, [7-12] carried out powerfl ow analysis of electrified tracks, wherein several new powerfl ow algorithms are introduced, based on which power supply capacity of traction power supply systems and voltage distribution in transmission systems can be analyzed. Another important research direction on trains, tramways, and buses is to apply regenerative braking energy and leverage the topology of the power supply system to enhance the system's efficiency and economic operation [13, 14]. These works focus on the energy consumption and control methods instead of the impact of moving vehicles on the voltage level and the stability of the distribution system.

While these aforementioned works also consider similar radial network configurations, no existing work studies the steady-state voltage profiles and the long-term voltage stability limits of electrified roads when vehicles are in motion. The traditional loads in power systems can vary the demands but resize atfi xed locations in the network diagram. WDC vehicles, on the other hand, may consume the same amount of power if they travel at a constant speed but change the positions over time. The advent of these new moving loads may alter the distribution system operation and thus calls for new studies on this topic. This paper, therefore, will construct a series of new patterns about the voltage profile along the electrified roads over time to study the voltage level change while the vehicles travel. This pattern can be used in voltage compensation design to maintain voltage levels across the network within a range specified by voltage regulation standards. Another important aspect of steady-state analysis considered in this work is the long-term voltage stability (LTVS) of the electrified road, which concerns the existence of a steady-state solution. While typical LTVS limits in power systems are regarded as the maximum loading level that the power line can support, the LTVS thresholds here can be quantified in terms of the maximum road length and the number of vehicles "safely" allowed to operate on the electrified roads equipped with 


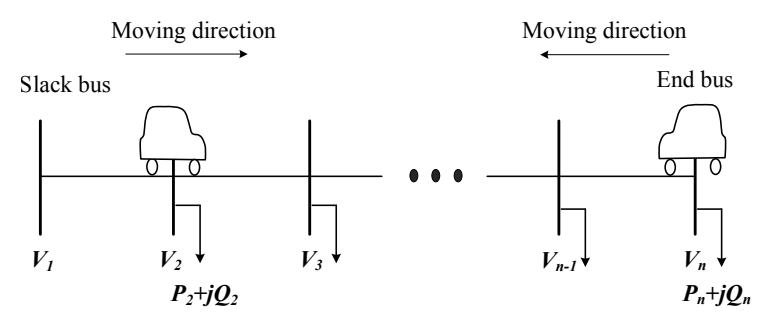

Figure 1: The diagram of simplified WDC system

WDC. We introduce a novel experimental setup below, relying on a widely used power software package in the community, i.e., MATPOWER [15], to perform our analysis.

\section{WDC EVS AND POWER FLOW STUDY}

\subsection{Wireless Dynamic Charging System}

This paper considers a simple dynamic charging EV system. The power source which is located at the beginning of the electrified road can be a generator or a coupling point connecting to a distribution grid. Then the power cable line under the electrified road connects power tracks, which are typically configured in series, to the power source. While a vehicle is moving over a power track, its battery can be recharged via the receiving resonator.

For simplicity, we assume that all EVs are homogeneous, and they travel at a constant speed with afi xed amount of power. While standard power system packages, such as MATPOWER [15], do not handle moving constant-power (PQ) buses, we propose to split the electrified road into equal-length segments marked withfictitious nodes such that, in each "snapshot" of powerfl ow study at a time step, thefl eet of vehicles arrives at a new set offi ctitious nodes. Such a new set offi ctitious nodes is then modeled as PQ buses, whereas, except for the reference bus, other nodes without EVs consume no power. As the EVs travel along the road, the set of PQ buses also changes. To understand how on-the-move vehicles affect the voltage profile along the road, we repetitively solve the power flow problem for such a series of time moments characterized by the set of PQ buses. Then the WDC system can be modeled, at one time-step, as a normal radial grid shown in Figure 1 and can be studied using power system packages. For the steady-state analysis, wefi nd the network voltage solution by solving the powerfl ow equations that are presented in Appendix A.

\section{VOLTAGE PROFILE}

The parameters of EVs and the WDC system are as following: active power per vehicle $\left(P_{E V}\right): 30 \mathrm{~kW}$; reactive power per vehicle $\left(Q_{E V}\right): 15 \mathrm{kVAr}$; resistance of the cable line: $0.568 \Omega / \mathrm{km}$; reactance of the cable line: $0.133 \Omega / \mathrm{km}$; on-board PV panel: $2 \mathrm{~kW}$; on-board capacitor: $100 \mathrm{kVAr}$; and thefi xed capacitor bank: $300 \mathrm{kVAr}$. As no common standard of EVs or WDC exists, we rely on several existing works in the literature that present the parameters of the used prototypes [16-20]. For the cable, we use a typical 6-kV distribution cable presented in [21]. In section 3, the road length and cable line are set to be $2 \mathrm{~km}$. Tenfi ctitious nodes are evenly distributed on the road. The voltage profiles along this electrified road are presented in the following sections, where we consider both one-way and two-way roads. Each moving direction has only one lane.

\subsection{One-way Road}

In this case, afl eet of two EVs moves from the slack bus to the end bus. At thefi rst time step, the two EVs start from node 1 and 2 .

3.1.1 DWC EVs system without voltage compensators. As shown in Figure 2, the voltage profiles, which collect the nodal voltages at the reference bus (node 1) to the end node (node 10), are constructed for nine different moments, called time steps, from $t_{1}$ to $t_{9}$. This set of voltage profiles resembles half-leaf veins. Each secondary vein is a voltage profile of a time step, while the main vein is the lower bound of all profiles, which will be defined later in this section. The nodal voltage reaches its minimum value at time step $t_{9}$ when vehicles reach the farthest nodes from the reference bus.

When the EVfl eet moves towards the end of the road, the nodal voltage decrease monotonically as the "travel" impedance between the slack bus and thefl eet's position increases so does the voltage drop. The lower bound of these voltage profiles can be characterized analytically as $V=V_{1}-I_{\max } Z$. Here, $V_{1}$ is the constant voltage of the reference node, and $I_{\max }$ is the largest effective current which corresponds to the heaviest loading condition happening at thefinal time step as the vehicles approach the end node. $Z$ is the effective "travel" impedance, which scales linearly as the vehicles travel and thus it is proportional to the bus number in our experiment. The lower bound is plotted as the dashed red line or the main vein of the half-leaf veins in Figure 2. The monotonic voltage profiles and the main vein can design voltage regulation.

Although the deployed constant-power load model is simple, it suffices to capture the most fundamental pattern of the voltage profile along the electrified road when the vehicles move. This simple model, however, can be extended to more complicated settings with charging efficiency, variable power consumption, and variable speeds. For instance, we can model the effective power of a vehicle as $P=P_{E V}-\triangle P$ and $Q=Q_{E V}-\triangle Q$ where $P_{E V}$ and $Q_{E V}$ are the base power consumption. We introduce $\Delta P$ and $\Delta Q$ to represent the power variation, for example, due to non-unity charging efficiency and variable speeds. Figure 3 plots three representative voltage profiles with error bars as the power variation can vary randomly within $50 \%$ of the base power level. The error bars become wider if the variation range gets larger. Moreover, for a given variation range, one still can construct the bounds of voltages by collating the corresponding extreme voltage values.

3.1.2 WDC EVs system with voltage compensators and PVs. In this experiment, $\mathrm{PV}$ panels or on-board capacitors are installed on each vehicle, whereas afi xed capacitor bank is set at bus 6-the middle of the road. For the on-board PV panel, the active power variation $\triangle P$ is used to represent the amount of $\mathrm{PV}$ injection. As $\mathrm{PV}$ produces more power, the effective power consumption of the vehicle becomes smaller. The on-board capacitor can be modeled in a similar way with the reactive power variation $\Delta Q$. However, unlike the onboard devices, thefi xed capacitor bank will not change the effective power consumption of the vehicles, but the self-susceptance of the associated node/bus where the capacitor bank is located [22].

In Figure 4, we plot several voltage profiles to show the effect of added PVs and voltage compensators. The lowest voltage profile represents the case without PVs and voltage compensators, while the most effective compensation method is using afi xed capacitor 
Steady-state Voltage Profile and Long-term Stability with Wireless Dynamic Charging e-Energy '19, June 25-28, 2019, Phoenix, AZ, USA

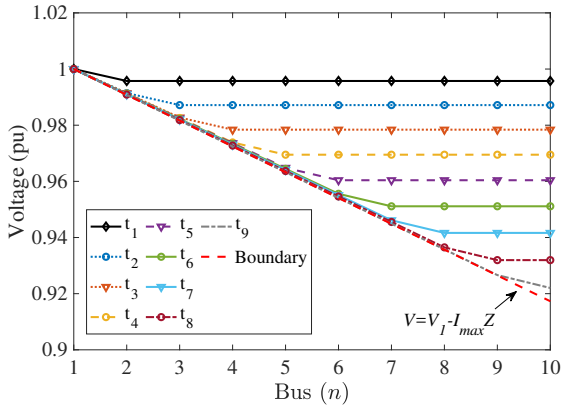

Figure 2: Voltage profile of WDC system (one direction)

bank. On-board capacitors affect the voltage of the bus where vehicles move over it, but thefi xed capacitor bank regulates voltage locally around bus 6 .

\subsection{Two-way Road}

In this section, a two-vehiclefl eet moves from the reference bus towards the end bus, while anotherfl eet of two vehicles moves from the opposite direction. At the beginning time, each pair of vehicles is located at thefi rst and the last two buses, respectively.

Figure 5 illustrates the voltage profiles when twofl eets of EVs move in two directions. Two interesting features of voltage profiles are observed in this case. First, the set of voltage profiles has a harp-like shape, as all individual voltage profiles lie inside two boundaries corresponding to two special time steps. One moment is when twofl eets of vehicles are located at their beginning positions; the other moment is when they meet near the middle of the road. However, if the number of vehicles in twofl eets is different, the minimum value of voltage will appear when thefl eet with more

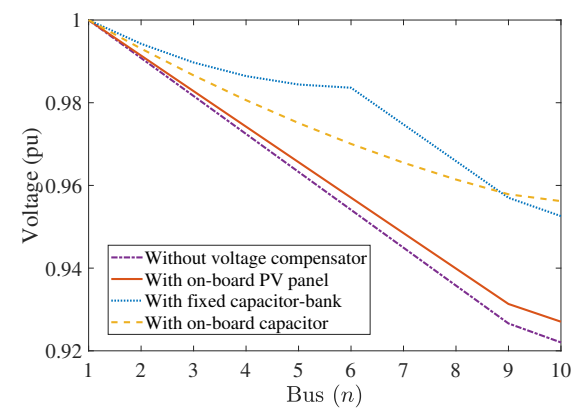

Figure 4: Voltage profile at thefi nal time step $t_{9}$

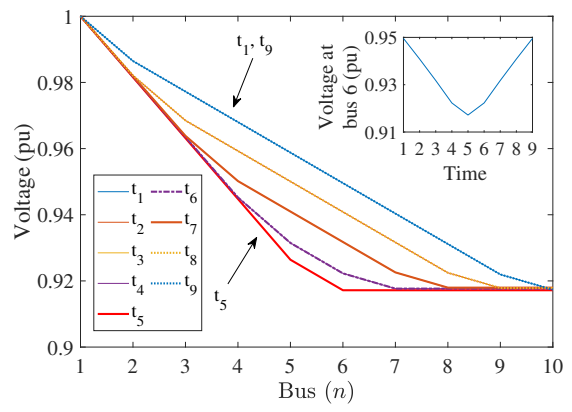

Figure 5: Voltage profile of WDC system (two directions)

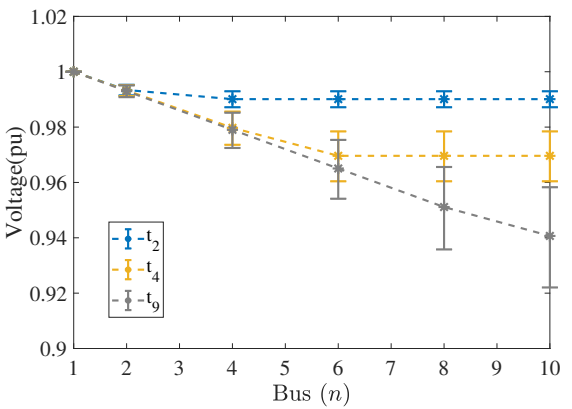

Figure 3: Voltage profile of WDC EV system considering more general settings (one moving direction)

vehicles reaches the end bus corresponding to their moving direction. The second feature is the repetition of voltage profiles, which creates voltage swings. As twofl eets of vehicles meet and continue following their original directions, some voltage profiles are overlapped. This explains why some moments such as $t_{1}$ and $t_{9}$ have the same voltage profile, or in other words, some voltage profiles are repeated. The insertedfi gure shows the voltagefl uctuation at bus 6 . Other buses also exhibit voltage swings but with different magnitudes. These voltage swings may cause problems related to power quality, voltage regulation, and power transfer losses.

\section{LONG-TERM VOLTAGE STABILITY}

In this section, we present a method to analyze the long-term voltage stability of the wireless dynamic charging EV system. Only a one-way road is considered in this case, but one can also extend the results to a two-way road easily. We rely on continuation power flow method (CPF) which has been used widely to quantify the maximum loadability corresponding to LTVS limits [23-25]. In CPF, one increases the injections gradually and then solves the resulting series of powerfl ow problems for the voltage levels. By collating the power injections and voltage solutions, one can construct nose curves and determine the loadability limit at the tip of the nose curve [26]. However, the CPF assumes that the positions of the injections arefi xed. The moving loads in the WDC problem, on the other hand, may maintain afi xed level of power consumption but change their locations during the experiment period. These moving loads, therefore, make CPF not directly applicable to an electrified road with WDC, except for a simple two-bus equivalent configuration as shown in Appendix B. For larger feeder networks with more EVs, one needs to apply CPF differently, as below.

\subsection{Maximum Road Length with Fixed EV Fleet}

Here, the size and power consumption of the EVfl eet arefi xed so that the long-term voltage stability becomes the problem of finding the maximum length of the road that corresponds to the most critical moment as vehicles reach the end of the road. The maximum length of the road can be determined, for afi xedfl eet of vehicles, by gradually increasing the length of the road until no voltage solution exists. This approach is known as nose curve scenario in the typical LTVS study, but here we do not aim to construct the conventional curve of the voltage magnitude and the active power, but the voltage-road length curves.

The detailed construction of such voltage-road length curves is as follows. First, wefi $\mathrm{x}$ the road length and then use continuation 


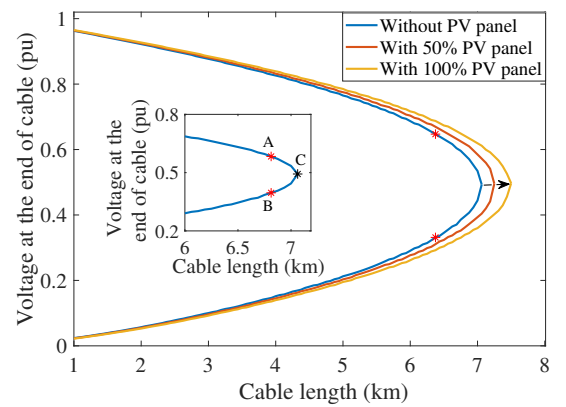

Figure 6: Maximum road length of WDC EV system. The black arrow indicates increasing PV installation.

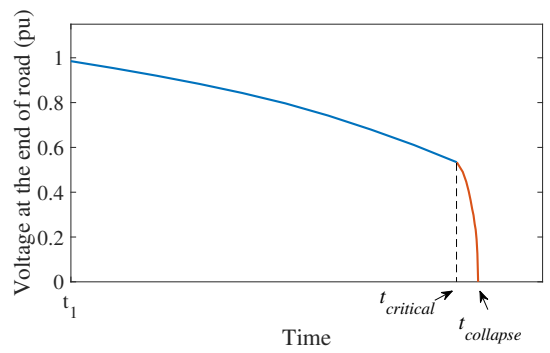

Figure 7: Voltage collapse phenomenon

powerfl ow tofi nd voltage solutions corresponding to the loading condition when all vehicles arrive at the endpoint [23]. If the road length is less than the critical length, one canfi nd two such voltage solutions, for example, the two red star-points in Figure 6. Then, we gradually increase the road length and collate all resulting voltage solutions to construct the voltage-road length curve. Continue this procedure until the tip $\mathrm{C}$ of the voltage-road length curve, where the pair of voltage solutions merges. Any road with a longer length than the critical length at point $C$ will result in no voltage equilibrium solutions when thefl eet of vehicles reaches its endpoint. This situation is regarded as a voltage collapse. Figure 6 depicts a number of voltage-road length curves from which the maximum road length can be determined. The blue curve presents the base case without PV panels installed on the EVs. With more added $\mathrm{PVs}$, the curves extend to the right that implies that the maximum length of the road can be larger. This effect makes sense from the steady-state study point of view. Another point to make is that the PV generation may vary depending on the weather so that the maximum length can differ from the base case. This effect needs to be addressed properly in the network design and operation to ensure safe operation of the road.

While most of the experimental results are intuitive, we discuss a non-intuitive result in this section. We consider a situation when the vehicles move beyond the maximum allowed length of the road then quickly "return back" within the safe length. Because the vehicle has returned to the safe length, one may expect the road is "safe". However, Figure 7 shows an unexpected outcome: the voltage collapses and goes to zero. While the complete mechanism of this collapse behavior is rather complicated [27], a simple explanation is that when the vehicles pass point $C$ towards point $B$ in the embeddedfi gure in Figure 6, the solution follows the lower branch of the nose curve which contains the segment CB. Even if the vehicles physically return to the safe length, the system voltage continues following the lower solution branch and does not return to point

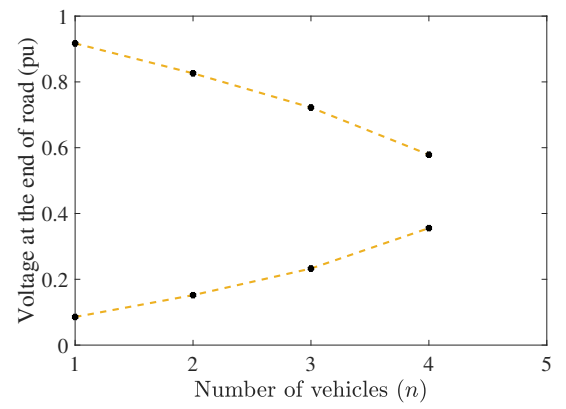

Figure 8: Maximum number of vehicles of WDC system

A, thenfi nally goes to zero. This collapse phenomenon develops in a short period from the moment the vehicles arrive at point $\mathrm{C}$ at $t_{\text {critical }}$ to $t_{\text {collapse }}$. The actual collapse event in practice has also been analysed in [28].

\subsection{Maximum Number of Vehicles on a Fixed Length Road}

This section focuses on the maximum number of EVs allowed to operate on afi xed length road. From Figure 2, we observe that the heaviest loading condition is when vehicles approach the end of the road. We can use the same method for constructing the voltage-road length curves in the previous section to build the voltage-number of EVs counterparts, by gradually increasing the number of EVs operating on the road. One difference, in this case, is that the number of EVs is not continuous but has discrete values. Figure 8 shows that at most four EVs can be allowed to operate simultaneously on a 4-km road. Varying segment sizes can change both the maximum length of the road and the maximum number of vehicles. In our experiments, we assume that all vehicle members in afl eet follow one another and that the gap between two consecutive members is equal to the segment size. If the segment size reduces, vehicle members run closer to each other and vice versa. Reducing the segment size will make thefl eet closer to the road end at thefinal time step and thus burden the electrified road further. Therefore, with a smaller segment size, the maximum road length and the number of vehicles allowed to operate need to be reduced.

\section{CONCLUSIONS AND FUTURE WORK}

In this paper, we study the effect of moving loads on the steadystate operation of a wireless dynamic charging system modeled as a one-feeder distribution gird. Voltage profiles of various operating conditions with PV panels and voltage compensators are constructed. We also present a method to apply continuation power flow for the LTVS of electrified roads to characterize the maximum length and maximum number of EVs that the road can accommodate. The new patterns of the distribution system's steady-state equilibrium regarding both the voltage and stability limits call for new research in this new paradigm. As this work deploys a rather simple model for the steady-state analysis of one electrified road, we plan to extend it to dynamic analysis with more mixed, detailed models of the distribution network in the future.

\section{ACKNOWLEDGMENTS}

Work of CW and HN was supported by NTU SUG. We thank Yashar Ghiassi for his shepherding. 


\section{REFERENCES}

[1] S. Jeong, Y. J. Jang, and D. Kum. Economic analysis of the dynamic charging electric vehicle. IEEE Transactions on Power Electronics, 30(11):6368-6377, Nov 2015

[2] Lefteris Karagiannopoulos. Electrified roads: Swedish project could slash cost of electric vehicles, 2018. Online.

[3] Daniel Boffey. World'sfi rst electrified road for charging vehicles opens in sweden, 2018. Online.

[4] I. Hwang, Y. J. Jang, Y. D. Ko, and M. S. Lee. System optimization for dynamic wireless charging electric vehicles operating in a multiple-route environment. IEEE Transactions on Intelligent Transportation Systems, 19(6):1709-1726, June 2018.

[5] J. M. Miller, P. T. Jones, J. Li, and O. C. Onar. Ornl experience and challenges facing dynamic wireless power charging of ev's. IEEE Circuits and Systems Magazine, 15(2):40-53, Secondquarter 2015.

[6] M. Yilmaz and P. T. Krein. Review of battery charger topologies, charging power levels, and infrastructure for plug-in electric and hybrid vehicles. IEEE Transactions on Power Electronics, 28(5):2151-2169, May 2013.

[7] Junqi Zhang, Mingli Wu, and Qiujiang Liu. A novel powerfl ow algorithm for traction power supply systems based on the thévenin equivalent. Energies, 11(1):126, 2018

[8] CJ Goodman and T Kulworawanichpong. Sequential linear powerfl ow solution for ac electric railway power supply systems. WIT Transactions on The Built Environment, 61, 2002.

[9] S. V. Raygani, A. Tahavorgar, S. S. Fazel, and B. Moaveni. Loadfl ow analysis and future development study for an ac electric railway. IET Electrical Systems in Transportation, 2(3):139-147, Sep. 2012.

[10] Wang Bin, Hu Haitao, Gao Shibin, and Han Xudong. Powerfl ow calculation for traction networks under regenerative braking condition based on locomotivetraction network coupling. Indonesian fournal of Electrical Engineering and Computer Science, 11(2):848-854, 2013.

[11] M Chymera, AC Renfrew, and M Barnes. Railway modelling for power quality analysis. WIT Transactions on The Built Environment, 88, 2006.

[12] Thanatchai Kulworawanichpong. Optimising AC electric railway powerfl ows with power electronic control. $\mathrm{PhD}$ thesis, University of Birmingham, 2004.

[13] Mikołaj Bartłomiejczyk and Marcin Połom. The impact of the overhead line's power supply system spatial differentiation on the energy consumption of trolleybus transport: planning and economic aspects. Transport, 32(1):1-12, 2017.

[14] Štefan Hamacek, Mikołaj Bartłomiejczyk, Roman Hrbáč, Stanislav Mišák, and Vítězslav Stỳskala. Energy recovery effectiveness in trolleybus transport. Electric Power Systems Research, 112:1-11, 2014

[15] Ray Daniel Zimmerman, Carlos Edmundo Murillo-Sanchez, and Robert John Thomas. Matpower: Steady-state operations, planning, and analysis tools for power systems research and education. IEEE Transactions on power systems, 26(1):12-19, 2011

[16] I Karakitsios, E Karfopoulos, and N Hatziargyriou. Impact of dynamic and static fast inductive charging of electric vehicles on the distribution network. Electric Power Systems Research, 140:107-115, 2016.

[17] J. G. Hayes, R. P. R. de Oliveira, S. Vaughan, and M. G. Egan. Simplified electric vehicle power train models and range estimation. In 2011 IEEE Vehicle Power and Propulsion Conference, pages 1-5, Sep. 2011.

[18] D Herron. Range confidence: Charge fast, drive far, with your electric car. Kättesaadav: https://greentransportation. info/ev-charging/range-confidence/chap8tech/evdc-fast-charging-standards-chademo-ccs-sae-combo-tesla-superchargeretc. html (15.05. 18), 2017.

[19] Jignesh Parmar. Defining size and location of capacitor in electrical system (1), 2016. Online.

[20] Junwei Lu and Jahangir Hossain. Vehicle-to-grid: Linking Electric Vehicles to the Smart Grid, volume 79. IET, 2015.

[21] Nexans. 6-36kv medium voltage underground power cables, 2010. Online.

[22] Ray D Zimmerman. Matpower 4.0 b4 user's manual. Power Syst Eng Res Cent, pages $1-105,2010$.

[23] Venkataramana Ajjarapu and Colin Christy. The continuation powerfl ow: a tool for steady state voltage stability analysis. IEEE transactions on Power Systems, 7(1):416-423, 1992.

[24] H. D. Nguyen, K. Dvijotham, S. Yu, and K. Turitsyn. A framework for robust long-term voltage stability of distribution systems. IEEE Transactions on Smart Grid, pages 1-1, 2018.

[25] Dhagash Mehta, Hung Dinh Nguyen, and Konstantin Turitsyn. Numerical polynomial homotopy continuation method to locate all the powerfl ow solutions IET Generation, Transmission \& Distribution, 10(12):2972-2980, 2016.

[26] S. Corsi and G. N. Taranto. Voltage instability - the different shapes of the "nose". In 2007 iREP Symposium - Bulk Power System Dynamics and Control - VII. Revitalizing Operational Reliability, pages 1-16, Aug 2007.

[27] Thierry Van Cutsem and Costas Vournas. Voltage stability of electric power systems. Springer Science \& Business Media, 2007.
[28] CD Vournas, GA Manos, John Kabouris, and Thierry Van Cutsem. Analysis of a voltage instability incident in the greek power system. In 2000 IEEE Power Engineering Society Winter Meeting. Conference Proceedings (Cat. No. 00CH37077), volume 2, pages 1483-1488. IEEE, 2000.

[29] Arthur R. Bergen and Vijay. Vittal. Power systems analysis /. Pearson Education, New Delhi, 2nd. edition, 2008.

\section{A POWER FLOW EQUATION}

The powerfl ow equation can be derived from Kirchhoff's circuit laws and used to describe the steady state condition of a power system. For bus $i$, the equation is as follows:

$$
P_{i}+j Q_{i}=\sum_{k=1}^{n} V_{i} V_{k}\left(\cos \theta_{i k}+j \sin \theta_{i k}\right)\left(G_{i k}-j B_{i k}\right)
$$

where $Y_{i k}=G_{i k}+j B_{i k}$ represent the admittance of branch $i k, v_{i}=$ $V_{i} \exp \left(j \theta_{i}\right)$ is the nodal complex voltage at bus $i$, and $\theta_{i k}=\theta_{i}-\theta_{k}$ denotes the angle difference between bus $i$ and bus $k$ [29].

For the steady-state analysis, we solve the set of powerfl ow equations (1) tofi nd the nodal voltages for given loading level $P_{i}$ and $Q_{i}, i=1, \ldots, n$. We then construct the voltage profile along the road by recording the solution voltages at different node locations while the vehicles are passing by.

\section{B CPF FOR A TWO-BUS SYSTEM}

To show that the conventional CPF can only handle moving loads in a two-bus system, we consider a toy example with one slack and one PQ bus connecting through a line with the impedance of $R+j X$. The two nodal voltages are $V_{1}$ and $V_{2}$. This configuration corresponds to the situation one vehicle travels in a one-way road. The consumption of the vehicle $P+j Q$ isfi xed, but the distance it travels increases over time; this can be captured by changing the line impedance duly. If the power factor and $X / R$ ratio are constant, a powerfl ow equation for this toy example can be written as follow

$$
U^{2}+\left(2 P R(\tan \phi \tan \theta+1)-\left(V_{1}\right)^{2}\right) U+(P R)^{2}(\sec \phi \sec \theta)^{2}=0
$$

where $\tan \phi=Q / P, \tan \theta=X / R, U=\left(V_{2}\right)^{2}$. Apparently, $R$ and $P$ play the same role in (2) so that we can change the power injection $P$ instead of the line resistance $R$ in CPF study. Unfortunately, this is not the case when we extend to more general network configurations. 